\title{
A Educação Física no Brasil em transição: perspectivas para a promoção da atividade física
}

\author{
Physical Education in a transitional Brazil: perspectives for physical activity \\ promotion
}

\author{
Filipe Ferreira da Costa ${ }^{1}$ \\ Leandro Martin Totaro Garcia² \\ Markus Vinicius Nahas ${ }^{3}$ \\ 1. Programa de Pós-Graduação em \\ Educação Física da Universidade \\ Federal de Santa Catarina (UFSC), \\ Florianópolis, SC, Brasil. \\ 2. Programa de Pós-Graduação em \\ Educação Física da Universidade \\ Federal de Santa Catarina (UFSC), \\ Florianópolis, SC, Brasil. \\ 3. Departamento de Educação Física da \\ Universidade Federal de Santa Catarina \\ (UFSC), Florianópolis, SC, Brasil.
}

\begin{abstract}
Resumo
O padrão de morbimortalidade no Brasil contemporâneo é caracterizado pelas altas prevalências de doenças crônicas não transmissíveis. O objetivo deste trabalho foi situar o papel da atividade física neste padrão de morbimortalidade, contextualizando o conceito de "Transição da Atividade Física" e discutindo a contribuição da Educação Física na promoção da atividade física e saúde. Parece evidente que houve redução no nível de atividade física nos domínios ocupacional, deslocamento e doméstico, em contrapartida à estabilização ou incremento no domínio do lazer. A inserção da Educação Física no Sistema Único de Saúde e as ações governamentais de promoção de atividade física são promissoras e necessitam de avaliação. A escola constitui contexto privilegiado para alcançar os jovens, além de seus familiares e dos moradores de seu entorno. Por fim, à Educação Física tem sido atribuída uma maior participação na promoção da saúde dos brasileiros, tanto por meio de ações no contexto da comunidade como na escola.
\end{abstract}

Palavras-chave: Atividade física; Promoção da saúde; Saúde pública; Saúde escolar.
ENDEREÇOPARA CORRESPONDÊNCIA

Filipe Ferreira da Costa

Rua José João Martendal, 385 - apto 303 Carvoeira - Florianópolis, SC 88040-420

filipefcosta_1@hotmail.com

\begin{abstract}
Morbidity and mortality pattern in Brazil is characterized by high prevalence of noncommunicable chronic diseases. The aim of this study was to situate the role of physical activity in this pattern, introducing the concept of "Physical Activity Transition" and discussing the contribution of Physical Education area in the physical activity and health promotion. It seems clear the reduction of physical activity levels in occupational, transportation, and domestic domains, in contrast to the stabilization or increase of leisure-time physical activity. Insertion of Physical Education area in the Unified Health System and the governmental policies to promote physical activity is promising and should be evaluated. School settings are of utmost importance to promote youth's health as well as of their families and the residents of its surroundings. To conclude, the Physical Education area has been an important agent for promotion of health among the Brazilian population, either through interventions in the community or school settings.
\end{abstract}

Keywords: Physical activity; Health promotion; Public health; School health.
- Recebido: $\quad$ 18/04/2011

- Re-submissão: 14/09/2011

- Aceito: $\quad$ 16/09/2011 


\section{INTRODUÇÃO}

O Brasil vem apresentando nas últimas décadas um quadro sóciossanitário complexo caracterizado pela coexistência de antigos problemas de saúde - típicos de países pouco desenvolvidos - com doenças do mundo moderno, denominadas de doenças crônicas não transmissíveis. As doenças não transmissíveis são responsáveis pelas maiores proporções de anos de vida perdidos por morte prematura, por anos de vida vividos com incapacidade e anos de vida perdidos ajustados por incapacidade ${ }^{1}$. Em 2008, as três causas específicas de óbitos mais frequentes no Brasil foram as doenças cerebrovasculares $(9,2 \%)$, as doenças isquêmicas do coração $(8,9 \%)$ e o diabetes mellitus $(4,7 \%)^{2}$. A obesidade, que em proporções epidêmicas está presente na maioria dos países desenvolvidos, acomete atualmente cerca de um em cada sete brasileiros. Ao considerar o Índice de Massa Corporal igual ou maior que $25 \mathrm{~kg} / \mathrm{m}^{2}$, estima-se que a metade dos adultos brasileiros encontra-se com excesso de peso ${ }^{3}$.

A ocorrência cada vez mais comum desses e de outros agravos crônicos não transmissíveis decorrem em grande parte das alterações na dinâmica social e econômica brasileira, que sofreu mudanças profundas a partir da segunda metade do século $X X$, seguindo a tendência mundial. Mudanças socioambientais como a urbanização, a melhoria das condições de moradia e saneamento, o acesso a serviços de saúde e a meios de diagnóstico, as mudanças econômicas, culturais e no estilo de vida das pessoas, entre outros aspectos, contribuíram para a caracterização atual do perfil de morbimortalidade do Brasil. Tais mudanças são apontadas na literatura como cruciais nos modelos de transição demográfica ${ }^{4}$, nutricional ${ }^{5}$ e epidemiológica ${ }^{6}$.
Nessa mesma perspectiva, em um recente artigo publicado por Katzmarzyk e Mason ${ }^{7}$, um modelo teórico foi proposto com o objetivo de abranger as mudanças nos padrões de atividade física e as suas consequências para a saúde e a expectativa de vida das pessoas. De acordo com esse modelo, o crescimento econômico, as mudanças no mundo do trabalho, na organização urbana das cidades, na percepção de violência das pessoas, entre outras transformações ocorridas na sociedade, vêm contribuindo para que um estilo de vida tradicional caminhe para um estilo de vida ocidental moderno. Se antes o cotidiano era caracterizado por atividades ocupacionais intensas, por um deslocamento ativo e pelo pouco tempo livre das pessoas, agora o trabalho é predominantemente sedentário, o deslocamento é motorizado e a maior parte do tempo livre disponível é dominada por atividades sedentárias. Esse modelo foi chamado de "Transição da Atividade Física" (Physical Activity Transition) e estaria intrinsecamente relacionado ao desenvolvimento de doenças crônicas como obesidade, hipertensão arterial, doença arterial coronariana, infarto do miocárdio, diabetes mellitus tipo 2, câncer, assim como à redução da aptidão física, com um impacto negativo na qualidade de vida das pessoas (Figura 1). Este cenário revelaria uma "má adaptação" ao estilo de vida sedentário contemporâneo, decorrente das mudanças econômicas e socioambientais citadas $^{8}$.

Diante das consequências do declínio nos níveis de atividade física da população e da importância no âmbito da saúde pública, pretendemos trazer à tona alguns indicadores da transição da atividade física no Brasil. Adicionalmente, considerando que a área acadêmica e profissional denominada Educação Física tem como seu principal objeto de investiga-
- Crescimento econômico

- Ambiente construído que conduz ao transporte motorizado

- Menor percepção de segurança no ambiente
- Urbanização

- Proliferação de equipamentos mecanizados

- Mudança no clima social

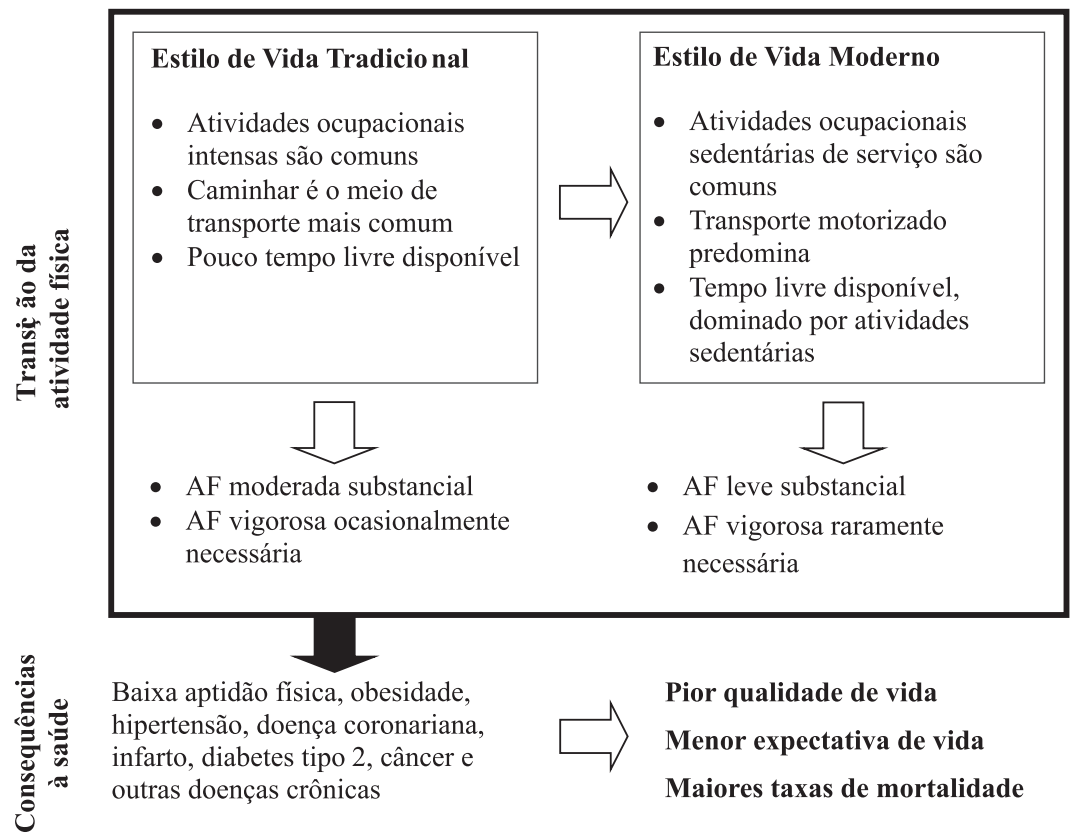


ção e de atuação a atividade física, entendida aqui como um comportamento humano complexo que possui atributos culturais, biológicos e psicossociais, este trabalho pretende discutir algumas das potencialidades desta área na promoção da saúde por meio da promoção da atividade física.

\section{A transição da atividade física no Brasil}

A inclusão da atividade física em inquéritos de saúde é um fato recente e resultados de levantamentos periódicos são ainda raros e disponíveis para poucos países, com destaque para os Estados Unidos, que desde 1986 coletam informações sobre atividade física que permitem análises de tendência. Segundo um estudo de revisão ${ }^{9}$, os poucos estudos disponíveis sugerem que a atividade física vem diminuindo no contexto do trabalho, enquanto, por outro lado, a participação em atividades físicas de lazer vem aumentando. Tais observações não podem ser extrapoladas para os países em desenvolvimento, uma vez que a maior parte destes estudos foi desenvolvida nos Estados Unidos e na Europa.

No Brasil, só a partir de 2006 a atividade física tem sido sistematicamente mensurada na população adulta das capitais por meio do sistema de Vigilância de Fatores de Risco e Proteção para Doenças Crônicas por Inquérito Telefônico (Vigitel) ${ }^{10}$. Tal fato limita a realização de uma investigação mais robusta da tendência temporal dos níveis de atividade física no contexto brasileiro. Portanto, procuramos caracterizar a seguir alguns dos fatores contribuintes para a transição da atividade física no Brasil, dando ênfase ao impacto que tiveram sobre os diferentes domínios da atividade física (ocupação, tarefas domésticas, deslocamento e lazer).

Em um passado relativamente recente, o Brasil possuía uma população eminentemente rural em que as atividades laborais relacionadas à produção agropecuária faziam parte do cotidiano da maioria das pessoas. Com o processo acelerado de urbanização na segunda metade do século passado, a população rural que em 1940 representava quase $70 \%$ da população do país, chegou em 2000 a menos de $20 \%{ }^{11}$. Com o fenômeno de urbanização e crescimento da economia, a ocupação laboral nos setores secundário (indústrias) e terciário (serviços) ganhou importância, e atividades ocupacionais que exigem grande esforço do trabalhador são cada vez menos comuns. Ao sintetizar os dados sobre ocupação de pessoas com 10 anos ou mais dos censos demográficos en- tre 1960 e 1991, Mendonça e Anjos ${ }^{12}$ revelaram uma redução importante na ocupação no setor da agricultura e extração, especialmente entre os homens, à custa do aumento nas ocupações relacionadas à indústria, ao comércio e aos serviços. Dados mais recentes confirmaram este padrão de mudança, e demonstram a manutenção da queda na proporção de empregados no setor da agricultura e aumento no setor de comércio e reparação entre os anos de 1992 e 2007 (Figura 2) ${ }^{11}$.

Essa tendência de mudança no padrão das principais ocupações no Brasil, em adição à crescente modernização de técnicas e equipamentos que reduzem o esforço físico do homem no trabalho, faz com que o gasto energético neste domínio represente cada vez menos no volume diário de atividade física do brasileiro. Apesar dessa tendência, vale lembrar que uma parcela dos trabalhadores brasileiros ainda possui atividades que demandam um alto gasto energético (ex.: limpeza urbana, serviço de correios, construção civil etc.).

Outro domínio da atividade física que sofreu profundas mudanças foi o deslocamento. $\mathrm{O}$ ato de caminhar ou pedalar como forma de deslocamento para o trabalho, resolver compromissos pessoais, ir ao mercado, visitar parentes ou ir à escola, deixou de ser um hábito para uma parcela importante dos brasileiros.

Infelizmente não existem dados nacionais que permitam descrever o declínio do deslocamento ativo entre os brasileiros ao longo do tempo. Contudo, ao assumir que parte deste declínio deve-se à crescente popularização da posse de veículos motorizados, podemos inferir sobre esta transição com base na frota de automóveis e motocicletas no Brasil. Segundo dados do Departamento Nacional de Trânsito ${ }^{13}$, em 1998 havia cerca de 17 milhões de automóveis registrados no Brasil comparados a quase 35 milhões no ano de 2009, um aumento de $100 \%$ em pouco mais de 10 anos (Figura 3). Um crescimento ainda maior ocorreu com a frota de motocicletas, que no mesmo período aumentou em seis vezes (Figura 4).

Ao relacionar estes dados com as estimativas da população brasileira, verificamos que em 1998 a relação era de cerca de um automóvel registrado para cada dez brasileiros, enquanto em 2009, a relação passou a ser de um automóvel para cada cinco pessoas (Figura 3). Em metrópoles como São Paulo, a relação chega a quase um carro registrado para cada dois habitantes. Já a variação na razão de pessoas por motocicleta foi ainda mais acentuada (Figura 4) ${ }^{13}$.

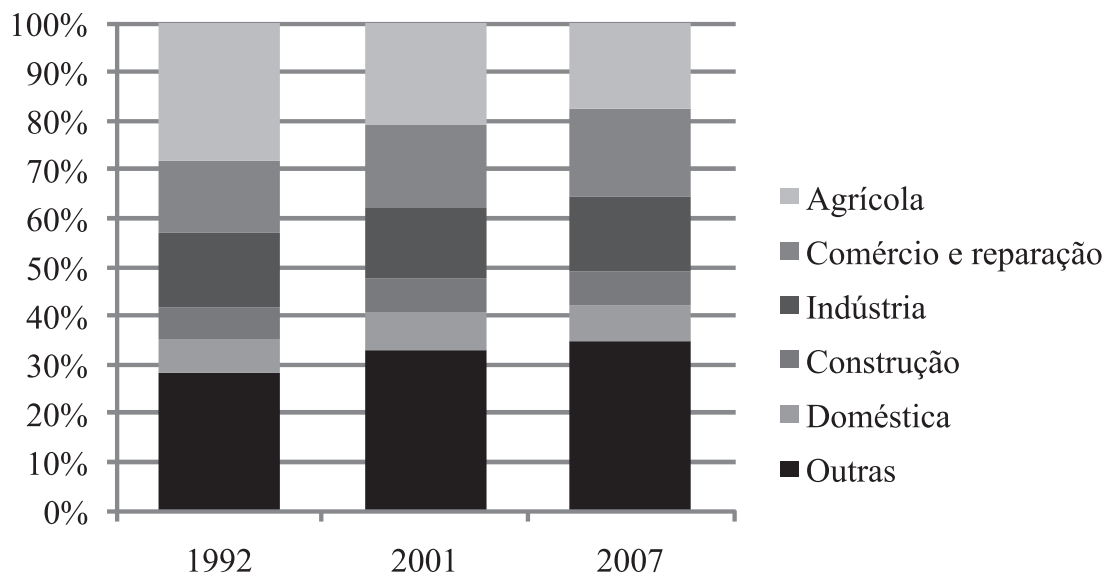

Ano 
Se por um lado o acesso a veículos automotores é cada vez mais comum, por outro podemos elencar aspectos ainda presentes em nossas cidades que atuam como barreiras importantes para o deslocamento ativo, como um sistema de transporte coletivo muitas vezes pouco eficaz e uma infraestrutura e um desenho urbano que desfavorecem o deslocamento ativo ${ }^{14}$, além de um ambiente social que desvaloriza tal hábito ${ }^{15}$

Os avanços tecnológicos, que reduziram o esforço na produção agrícola e de bens materiais, também tiveram seu impacto nos lares dos brasileiros. As atividades relacionadas à limpeza e manutenção da casa certamente demandam menos tempo e esforço das pessoas hoje do que há trinta ou quarenta anos. Atualmente existe uma diversidade de recursos como máquinas de lavar roupa e louças, aspirador de pó e outros eletrodomésticos que facilitam o trabalho doméstico e que são acessíveis a um grupo cada vez maior de brasileiros. Segundo dados da Pesquisa Nacional por Amostra de Domicílios ${ }^{16}$, a existência de máquina de lavar roupa nos domicílios brasileiros passou de 24,3\% em 1993 para 44\% em 2009 - um aumento de $81 \%$. Embora este maior acesso possa representar uma redução do gasto energético em tarefas domésticas, uma parcela expressiva de mulheres ainda relata serem responsáveis pela limpeza pesada de suas casas - 71,4\% contra $21,7 \%$ entre os homens - segundo dados do Vigitel ${ }^{17}$.
O quarto e talvez o mais importante contexto de prática da atividade física na atualidade é o do lazer. A prática de atividade física no tempo livre ganhou importância uma vez que este período passou a ser, para muitas pessoas, o único momento em que podem movimentar-se em níveis mais elevados de intensidade, frequência e duração.

$O$ primeiro estudo em escala nacional que avaliou este comportamento foi a Pesquisa sobre Padrões de Vida realizada pelo IBGE nos anos de 1996-97. Neste estudo, 13\% dos entrevistados relataram fazer exercícios ou esportes por pelo menos 30 minutos em um ou mais dias da semana, enquanto apenas 3,3\% relataram realizar alguma atividade por pelo menos 30 minutos em cinco ou mais dias da semana ${ }^{18}$. Recentemente, dados do Vigitel demonstraram que a prevalência de indivíduos que relataram praticar algum esporte ou exercício físico estabilizou entre os anos de $2006(14,9 \%)^{19}$ e 2009 $(14,7 \%)^{10}$.

Outros dois estudos sobre tendência temporal foram realizados recentemente em diferentes regiões do Brasil. Em Pelotas (RS), observou-se que a prevalência de adultos que não atingiam 150 minutos de atividades físicas moderadas a vigorosas passou de $41 \%$ em 2002 para 52\% em 2007, sendo que o aumento foi maior entre os mais pobres ${ }^{20}$. É importante ressaltar que a atividade física de lazer tem sido consistentemente associada a uma maior renda e escolaridade ${ }^{17,18}$, denun-
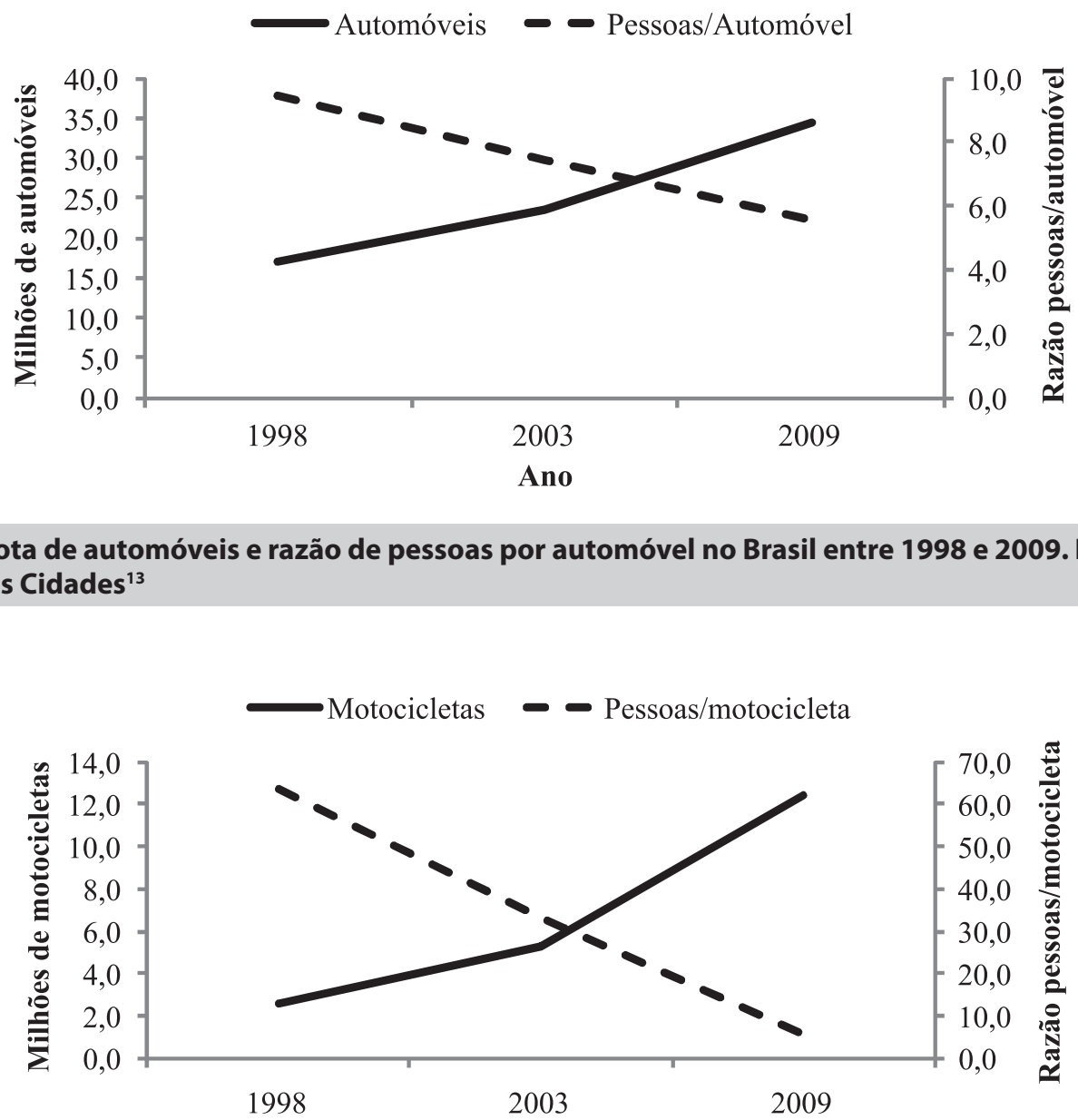

Ano 
ciando que, muitas vezes, tal comportamento é um privilégio de grupos populacionais que gozam de melhores condições socioeconômicas. Fatores como uma carga de trabalho exaustiva, o menor acesso a espaços públicos e privados de lazer e menor exposição a normas e ações que apoiem a prática de atividades físicas podem constituir barreiras para a prática de atividade física no lazer que afetam em maior grau os indivíduos de nível socioeconômico menos favorecido ${ }^{21}$.

O segundo estudo aconteceu no estado de São Paulo, demonstrando resultados contrários aos vistos até aqui. A prevalência de adultos que não atingiam 150 minutos de atividades físicas passou de 44\% em 2002 para 12\% em 2008. Além disso, a prevalência de pessoas que relataram não praticar qualquer atividade física (caminhadas e atividades moderadas a vigorosas) reduziu de $10 \%$ a $3 \%$ no mesmo período ${ }^{22}$.

Embora os estudos disponíveis não permitam estabelecer de maneira clara qual é a tendência do nível de atividade física no Brasil ao longo do tempo, os dados de outros países e aqueles existentes para nossa população sugerem que estamos passando por um período de transição. Enquanto no passado o padrão era caracterizado predominantemente por ocupações que exigiam maior esforço físico, por um deslocamento realizado caminhando ou pedalando, pela realização de tarefas domésticas pelas próprias pessoas e sem auxílio de equipamentos modernos, assistimos nas últimas décadas a um rápido desenvolvimento econômico no Brasil, que vem popularizando o acesso a veículos automotores, aos equipamentos que facilitam e agilizam as tarefas domésticas e no trabalho, além de uma maior proporção de trabalhadores no setor de serviços. Dados do Vigitel de 2006 a 2009 sobre a inatividade física nos quatro domínios (lazer, trabalho, deslocamento e limpeza doméstica) ilustram essa tendência recente (Figura 5) 10,19,23,24. Tais mudanças parecem ser irreversíveis, e indicam a necessidade de ações intersetoriais e multidisciplinares para promover um estilo de vida ativo para a população brasileira.

\section{A contribuição da Educação Física}

As evidências disponíveis sobre os efeitos benéficos da atividade física nos níveis de saúde, e, por outro lado, o reconhecimento de que o sedentarismo é um fator de risco importante para o desenvolvimento de diversas doenças crônicas não transmissíveis mais prevalentes na sociedade moderna, vêm aproximando a Educação Física da área da saúde pública. Como consequência, a investigação do nível de atividade física de grupos populacionais passa a ser de interesse não apenas da área acadêmica e profissional da Educação Física. Cada vez mais a área da saúde pública se interessa por tais investigações, fato que pode ser constatado no número crescente de publicações nos periódicos da área da saúde coletiva que tiveram a variável atividade física como desfecho principal ou como variável explanatória ${ }^{25}$, ou ainda na participação crescente de professores de Educação Física em programas de pós-graduação de outras áreas da saúde.

\section{Educação Física e Saúde Pública}

A promoção da saúde, aqui entendida como uma estratégia de produção social de saúde, abrange ações intersetoriais voltadas para os múltiplos determinantes do processo saúde-doença (condições adequadas de habitação, trabalho e alimentação, acesso aos serviços de saúde, educação, cultura, lazer, entre outros) ${ }^{26}$. A promoção da atividade física deve, portanto, ser entendida como uma das possibilidades de se promover melhores níveis de saúde, possuindo implicações diversas na vida das pessoas (melhora da capacidade funcional e da qualidade de vida, redução de riscos à saúde, interação social etc.) e no ambiente físico que as cercam (melhoria de espaços públicos, mobilidade urbana, segurança etc.).

Sob essa perspectiva, a promoção de atividade física tem destaque na Política Nacional de Promoção da Saúde promovida desde 2006 pelo Ministério da Saúde, com prioridade para quatro áreas: ações na rede básica de saúde e na comunidade; ações de aconselhamento e divulgação; ações intersetoriais e de mobilização de parceiros; e ações de monitoramento e avaliação ${ }^{26}$. Com a criação dos Núcleos de Atenção à Saúde da Família no âmbito da Atenção Básica em 2008, o profissional de Educação Física passou a ser legalmente habilitado para compor o quadro de profissionais atuantes na atenção básica à saúde. Neste contexto, as ações de atividade física foram entendidas como estratégicas para a promoção da qualidade de vida e prevenção do adoecimento da população adstrita nas unidades básicas de saúde ${ }^{27}$.

Embora na prática o profissional de Educação Física já esteja atuando no âmbito da Atenção Básica em muitas localidades do Brasil, poucas propostas de intervenções foram sistematizadas e disponibilizadas na literatura nacional. A

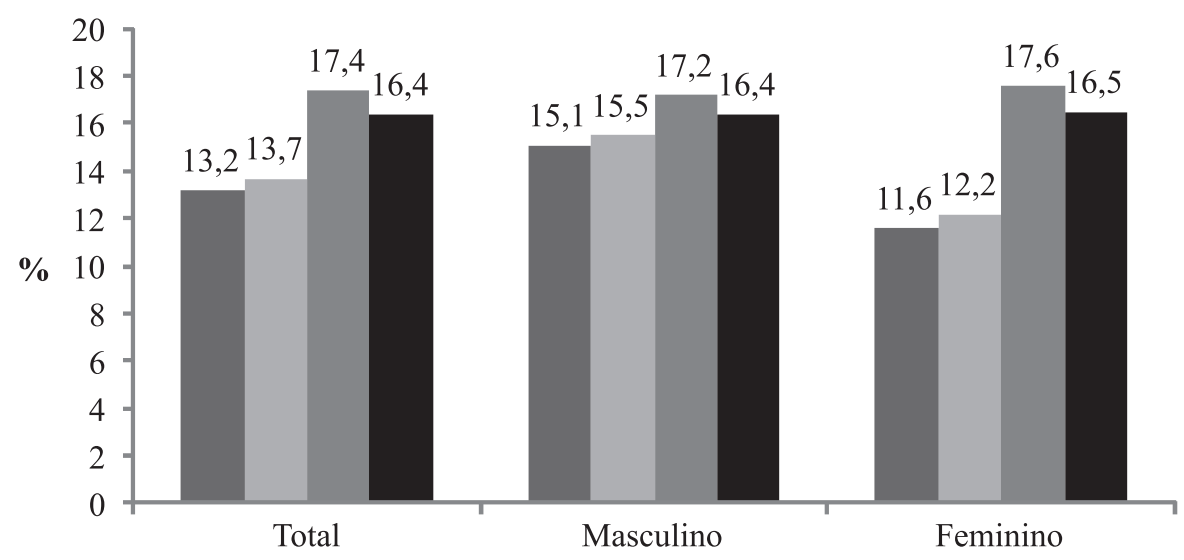


análise e avaliação destas práticas merecem especial atenção, uma vez que as competências para a atuação profissional no âmbito do Sistema Único de Saúde ainda não foram estabelecidas nos currículos dos cursos superiores em Educação Física.

Em 2007, Almeida realizou um estudo no qual desenvolveu, implantou e avaliou um programa de orientação de atividade física para a promoção da saúde em adultos aparentemente saudáveis, no âmbito do Programa de Saúde da Família 28. O Modelo Transteorético e a Teoria Educacional de Paulo Freire foram utilizados, e os resultados indicaram que no grupo experimental houve mudanças positivas na percepção de saúde, no nível de atividade física habitual e nos estágios de mudança de comportamento, além de diminuição na percepção da barreira "falta de conhecimento e/ou orientação para atividade física" 28 .

No município de São Paulo-SP, uma experiência de atuação profissional em um Centro de Saúde foi documentada por Freitas ${ }^{29}$. Por meio de estratégias que promoviam a participação e autonomia dos usuários, conteúdos diversos relacionados à atividade física e ao cuidado com a saúde foram desenvolvidos em aulas dentro da unidade de saúde e em seu entorno. O relato discute a inserção do profissional no sistema de saúde, e aponta a necessidade de articulação dos elementos constituintes deste campo de atuação, a saber, a contribuição inerente da área da Educação Física, o serviço de saúde, representado pela unidade de saúde, e os usuários, que constituem o público alvo das ações ${ }^{29}$.

É imprescindível a proposição e avaliação de formas de atuação do profissional de Educação Física no âmbito da Atenção Básica, em especial no contexto da Estratégia de Saúde da Família. Tais investigações podem subsidiar discussões sobre as competências profissionais para atuar no sistema público de saúde e conduzir a uma reformulação dos currículos de ensino superior em Educação Física.

Outro caminho para a contribuição da Educação Física no âmbito da saúde pública são os programas comunitários de promoção da atividade física, desenvolvidos em nível municipal e com a possibilidade de participação da esfera federal no financiamento dos projetos e programas. Algumas experiências têm sido documentadas ${ }^{30-34}$ e os primeiros estudos de avaliação foram recentemente publicados na literatura. Destacam-se os programas Academia da Cidade desenvolvido em Recife e a experiência de Curitiba (Curitibativa). O primeiro abrange atividades orientadas por profissionais de Educação Física que incluem avaliações físicas, aulas de ginástica e dança, grupos de caminhada entre outras atividades realizadas em praças e parques da cidade ${ }^{32}$. O segundo é caracterizado por ações e programas intersetoriais que envolvem diferentes setores da prefeitura, incluindo tanto estratégias voltadas para a modificação do ambiente (praças, parques e ciclovias) quanto àquelas com ênfase na educação para a saúde e na prática de atividades físicas (avaliação física, orientação e prescrição de atividades e caminhadas) ${ }^{33}$.

Mesmo considerando a importância e a necessidade de ações voltadas para o indivíduo, ou seja, das estratégias que focam na educação para a saúde e na oferta de práticas orientadas, a promoção da atividade física, e por consequência da saúde, envolve estratégias e ações diversificadas que podem ser mais ou menos abrangentes. No Brasil, é crescente o interesse em investigar os fatores ambientais relacionados à prática de atividade física, entendidos como tudo aquilo que foge ao escopo individual de análise (por exemplo, suporte social, desenho urbano, segurança pública e equipamentos públicos de lazer). Dados do Vigitel mostraram que a existência de lo- cais públicos de lazer foi associada à prática atividade física de lazer em adultos brasileiros ${ }^{35}$. Em um estudo realizado em São Paulo foi encontrado que a existência de estruturas como academias, praças, quadras, postos de saúde e bancos, assim como o suporte social e uma boa percepção de segurança, foram associadas a atividades físicas de lazer em idosos ${ }^{36}$. A percepção de falta de espaço, de opções de atividade e de equipamentos estiveram associadas a não utilização dos parques para a prática de atividades físicas entre rapazes e moças de Curitiba ${ }^{37}$. Tais evidências, embora baseadas em estudos transversais, sugerem que atributos do ambiente físico e social devem ser considerados nas políticas de saúde pública destinadas à promoção da atividade física.

\section{A escola como contexto privilegiado}

À escola são atribuídas funções sociais abrangentes direcionadas à educação para a vida em seus diferentes domínios. Nesta perspectiva, com uma abordagem integral e visando o desenvolvimento humano sustentável, a Iniciativa Regional Escolas Promotoras de Saúde aponta como um dos seus componentes a provisão de serviços de saúde, nutrição saudável e vida ativa, juntamente com a educação para a saúde e a criação e manutenção de ambientes físicos e psicossociais saudáveis ${ }^{38}$. Dando suporte a esta iniciativa, as evidências sugerem que intervenções no ambiente escolar são efetivas para elevar os níveis de atividade física ${ }^{39,40}$, assim como para promover uma dieta mais saudável entre os escolares ${ }^{41,42}$. O potencial de influência da escola na atividade física de crianças e adolescentes fica evidente quando consideramos que eles passam boa parte de seus dias de semana na escola, e podem ser fisicamente ativos antes e depois das aulas, durante os intervalos e nas aulas de Educação Física. Além do mais, os processos didático-pedagógicos desenvolvidos no contexto escolar, como na disciplina de Educação Física, por exemplo, podem contribuir para que os jovens adotem um estilo de vida ativo e saudável no contexto extraescolar.

Por tratar com o corpo em movimento, a Educação Física escolar é uma disciplina privilegiada para promover a atividade física entre os jovens. Apesar deste reconhecido potencial, assistimos hoje a uma desvalorização desta disciplina curricular no contexto da escola. Segundo dados da Pesquisa Nacional de Saúde do Escolar, apenas $49 \%$ dos escolares do $9^{\circ}$ ano frequentaram aulas de Educação Física duas vezes ou mais nos setes dias anteriores, enquanto que, por outro lado, 20\% relataram não ter tido nenhuma aula ${ }^{43}$. Tal realidade parece ocorrer em muitos países, e está intimamente relacionada à redução do tempo curricular da Educação Física em detrimento do aumento de outras atividades e disciplinas voltadas para o rendimento acadêmico ${ }^{44}$.

Mesmo considerando que as atividades físicas realizadas nas aulas de Educação Física componham o nível de atividade física total de crianças e adolescentes, seria ingênuo acreditar que as aulas poderiam "compensar" os baixos níveis de atividade física encontrados neste grupo etário. Pelo contrário, evidências sugerem que o tempo real em movimento nas aulas é baixo e não atinge níveis de intensidade de esforço relacionados à saúde ${ }^{45,46}$. Por isso, desde a década de 90 algumas propostas e sugestões curriculares de Educação Física têm abordado a promoção e a educação para um estilo de vida ativo e saudável ao longo da vida, que vão além dos objetivos imediatos da prática de atividade física ${ }^{47-49}$. Tais propostas apresentam alguns pontos em comum, destacados aqui pelo seu potencial para promover a atividade física, e, por conseguinte, a saúde de jovens: a) as experiências motoras nas 
aulas de Educação Física devem ser prazerosas, favorecendo a autoestima e uma atitude positiva do jovem em relação à atividade física; b) os objetivos e conteúdos devem ser progressivos e sequenciais, garantindo que as aulas respeitem o nível de desenvolvimento dos jovens em cada nível de ensino e não sejam repetitivos; e c) conceitos e informações sobre atividade física, aptidão física e saúde devem ser explorados com maior ênfase entre os adolescentes mais velhos, favorecendo a autonomia para a adoção de um estilo de vida ativo e saudável. Outra ação que poderia contribuir na promoção da atividade física é a inclusão de mais aulas de Educação Física, de boa qualidade, nos currículos das escolas, contrapondo a atual tendência de redução do número de aulas.

Dado que os níveis de atividade física de jovens e adultos, assim como a saúde em seu conceito mais amplo, são determinados por múltiplos fatores, adicionamos aqui a necessidade de incluir ações didático-pedagógicas que favoreçam a cidadania e o emponderamento dos jovens em busca de meIhores condições e oportunidades para a prática de atividade física, como as questões relacionadas à segurança pública e a criação e manutenção de ambientes saudáveis - por exemplo, a própria escola, praças, parques, quadras esportivas, calçadas etc.

Somado ao potencial da disciplina de Educação Física, o ambiente escolar por si só parece influenciar os níveis de atividade física dos jovens. A existência de equipamentos (quadras com traves, redes, demarcações etc.) e playgrounds na escola está associada a maiores níveis de atividade física mensurada tanto por observação direta ${ }^{50}$ quanto por acelerômetros ${ }^{51,52}$. Um estudo recente validou um instrumento para avaliar o suporte físico das escolas para caminhar, pedalar, brincar, jogar entre outras atividades realizadas pelas crianças, e verificou que os escores apresentados pelas escolas (maior vs. menor quintil) identificaram diferenças nos níveis de atividade física das crianças ${ }^{52}$.

Corroborando o que foi exposto, Ribeiro e colaboradores, após detalhada revisão sobre as intervenções existentes na América Latina, recomendaram quatro estratégias para a promoção de atividades físicas no ambiente escolar: mudanças curriculares nas aulas de Educação Física; infraestrutura adequada e treinamento dos funcionários da escola; provisão de equipamentos e materiais; e o direcionamento das ações de acordo com as características da população-alvo ${ }^{53}$.

Nota-se que as três últimas recomendações tornam a escola um ambiente de promoção da atividade física não somente entre os alunos, mas potencialmente para seus familiares, para os trabalhadores da escola e para a comunidade em que está inserida. Esta é uma possibilidade ainda pouco explorada e avaliada no país. Um exemplo são os Centros Educacionais Unificados em São Paulo-SP, que oportunizam aos moradores dos bairros assistidos o acesso a equipamentos públicos de lazer, cultura, tecnologia e práticas esportivas, inclusive nos finais de semana.

Em resumo, a escola pode contribuir de forma substancial para a promoção da atividade física entre os jovens sob duas principais perspectivas. Uma delas é pela criação de ambientes físicos e sociais propícios para a atividade física no tempo em que estão na escola (antes e depois das aulas, no recreio, em atividades extras no contraturno escolar etc.). A outra seria por meio de ações didático-pedagógicas desenvolvidas pelas disciplinas curriculares. Nesta perspectiva, a Educação Física teria papel fundamental pelo seu potencial de promover experiências, conhecimentos e atitudes positivas em relação à atividade física e saúde. Além disso, é necessário explorar o potencial das escolas para promover a prática de atividades físicas na comunidade em que estão inseridas, assumindo um novo papel na saúde pública.

\section{CONSIDERAÇÕES FINAIS}

Não existem dúvidas que o Brasil, assim como a maioria dos países, vem passando por mudanças econômicas e sociais que são determinantes das condições e modos de vida das pessoas. Tais condições e modos de vida são, por conseguinte, determinantes dos níveis de saúde e morbimortalidade da população. Apresentamos e discutimos brevemente neste trabalho alguns dos indicadores do impacto que essas mudanças tiveram sobre os níveis de atividade física da população brasileira, resgatando o conceito de "transição da atividade física". Embora os dados disponíveis não permitissem fazer análises de tendência temporal, pode-se inferir que os níveis de atividade física nos domínios do trabalho, deslocamento e doméstico parecem ter reduzido ao longo do tempo, enquanto as atividades no domínio do lazer tenderam a se estabilizar.

Em um segundo momento, discutimos o potencial que a área acadêmica e profissional da Educação Física possui na promoção da atividade física e saúde da população brasileira. Destacamos a recente inserção do profissional de Educação Física no SUS, mais especificamente na Atenção Básica, além da criação e ampliação de programas de promoção de atividade física nos municípios brasileiros. Apesar desta inserção crescente e da expansão dos programas, ainda são escassos os relatos e avaliações destas experiências na literatura científica. Somado a isto, verifica-se que ainda não existe uma discussão ampliada sobre uma formação acadêmica e profissional que seja coerente com a inserção da área nos serviços de saúde pública. Destacamos também a escola como contexto privilegiado, tanto para as ações voltadas à criação de um ambiente propício para a prática de atividade física quanto às ações didático-pedagógicas nas aulas de Educação Física, além de sua potencialidade como polo de promoção dentro das comunidades.

Diante disso, pelo seu objeto de investigação e intervenção profissional, à Educação Física tem sido atribuída uma maior participação na promoção da saúde da população brasileira. Discussões sobre a formação acadêmica e profissional, assim como a melhor divulgação das experiências já desenvolvidas, são necessárias para fundamentar e delinear novas perspectivas de promoção da atividade física e da saúde.

\section{Fontes de financiamento ou conflitos de interesse} Não houve.

\section{Contribuição dos autores}

Filipe Costa sugeriu a elaboração do manuscrito, além de redigi-lo e revisá-lo. Leandro Garcia redigiu e revisou o manuscrito. Markus Nahas contribuiu de forma importante na revisão crítica do texto.

\section{REFERÊNCIAS}

1. Schramm JM, Oliveira AF, Leite IC et al. Transição epidemiológica e o estudo de carga de doença no Brasil. Cien Saude Colet 2004;9:897-908.

2. Ministério da Saúde (BR). Saúde Brasil 2009: uma análise da situação de saúde e da agenda nacional e internacional de prioridades em saúde. Brasília: Ministério da Saúde; 2010.

3. Instituto Brasileiro de Geografia e Estatística. Pesquisa de orçamentos familiares (POF) 2008-2009: antropometria e estado nutricional de crianças, adolescentes e adultos no Brasil. Rio de Janeiro: Instituto Brasileiro de Geografia e Estatística; 2010

4. Wong LL, Carvalho JA. O rápido processo de envelhecimento populacional do Brasil: sérios desafios para as políticas públicas. Rev Bras Estud Popul 
2006;23:5-26.

5. Popkin BM. The nutrition transition in low-income countries: an emerging crisis. Nutr Rev 1994;52:285-98.

6. Prata PR. A transição epidemiológica no Brasil. Cad Saude Publica 1992;8:16875.

7. Katzmarzyk PT, Mason C. The physical activity transition. J Phys Act Health 2009;6:269-80

8. Malina RM, Little BB. Physical activity: the present in the context of the past. Am J Hum Biol 2008:20:373-91.

9. Knuth AG, Hallal PC. Temporal trends in physical activity: a systematic review. J Phys Act Health 2009;6:548-59.

10. Ministério da Saúde (BR). Secretaria de Vigilância em Saúde, Secretaria de Gestão Estratégica e Participativa. Vigitel Brasil 2009: vigilância de fatores de risco e proteção para doenças crônicas por inquérito telefônico. Brasília: Ministério da Saúde; 2010.

11. Instituto Brasileiro de Geografia e Estatística. Séries estatísticas \& séries históricas. Rio de Janeiro: Instituto Brasileiro de Geografia e Estatística. http://www. ibge.gov.br/series_estatisticas. Acessado em 20 de novembro de 2010.

12. Mendonça $C P$, Anjos LA. Aspectos das práticas alimentares e da atividade física como determinantes do crescimento do sobrepeso/obesidade no Brasil. Cad Saude Publica 2004;20:698-709.

13. Ministério das Cidades, Departamento Nacional de Trânsito (BR). Frota de veículos, por tipo e com placa, segundo as Grandes Regiões e Unidades da Federação. Brasília: Ministério das Cidades. http://www.denatran.gov.br/frota. htm. Acessado em 20 de novembro de 2010.

14. Frank L, Kavage S. A national plan for physical activity: the enabling role of the built environment. J Phys Act Health 2009;6(Suppl 2):S186-95.

15. Wendel-Vos W, Droomers M, Kremers S, Brug J, Van Lenthe F. Potential environmental determinants of physical activity in adults: a systematic review. Obes Rev 2007;8:425-40

16. Instituto Brasileiro de Geografia e Estatística. Pesquisa nacional por amostra de domicílios. Rio de Janeiro: Instituto Brasileiro de Geografia e Estatística. http://www.sidra.ibge.gov.br/pnad/. Acessado em 15 de novembro de 2010.

17. Malta DC, Moura EC, Castro AM et al. Padrão de atividade física em adultos brasileiros: resultados de um inquérito por entrevistas telefônicas, 2006. Epidemiol Serv Saude 2009;18:7-16.

18. Monteiro CA, Conde WL, Matsudo SM et al. A descriptive epidemiology of leisure-time physical activity in Brazil, 1996-1997. Rev Panam Salud Publica 2003;14:246-54

19. Ministério da Saúde (BR). Vigitel Brasil 2006: vigilância de fatores de risco e proteção para doenças crônicas por inquérito telefônico. Brasília: Ministério da Saúde; 2007.

20. Knuth AG, Bacchieri G, Victora CG, Hallal PC. Changes in physical activity among Brazilian adults over a 5-year period. J Epidemiol Community Health 2010;64:591-5.

21. Lee RE, Cubbin C. Striding toward social justice: the ecologic milieu of physical activity. Exerc Sport Sci Rev 2009;37:10-7.

22. Matsudo VK, Matsudo SM, Araújo TL et al. Time trends in physical activity in the State of São Paulo, Brazil: 2002-2008. Med Sci Sports Exerc 2010;42:2231-6.

23. Ministério da Saúde (BR). Vigitel Brasil 2007: vigilância de fatores de risco e proteção para doenças crônicas por inquérito telefônico. Brasília: Ministério da Saúde; 2008

24. Ministério da Saúde (BR). Vigitel Brasil 2008: vigilância de fatores de risco e proteção para doenças crônicas por inquérito telefônico. Brasília: Ministério da Saúde; 2009

25. Hallal PC, Dumith SC, Bastos JP et al. Evolution of the epidemiological research on physical activity in Brazil: a systematic review. Rev Saude Publica 2007:41:453-60

26. Ministério da Saúde (BR). Política nacional de promoção da saúde. Brasília: Ministério da Saúde; 2006.

27. Ministério da Saúde (BR). Portaria n 154, de 24 de janeiro de 2008. Cria os Núcleos de Apoio à Saúde da Família - NASF.

28. Almeida MG. Orientação de atividade física em programa de Saúde da Família: uma proposta de ação (dissertação de mestrado). Florianópolis: Universidade Federal de Santa Catarina, 2007
29. Freitas FF. A educação física no serviço público de saúde. São Paulo: Hucitec 2007.

30. Guarda FR, Silva RN, Marcondes MM, Marcondes AC, Almeida TA. Do diagnóstico à ação: programa Se Bole Olinda: estratégia intersetorial de promoção da saúde através da atividade física. Rev Bras Ativ Fis Saude 2009;14:206-10.

31. Mendonça $B C$, Toscano JJ, Oliveira AC. Do diagnóstico à ação: experiências em promoção da atividade física programa Academia da Cidade Aracaju: promovendo saúde por meio da atividade física. Rev Bras Ativ Fis Saude 2009;14:211

32. Simões EJ, Hallal P, Pratt M et al. Effects of a community-based, professionally supervised intervention on physical activity levels among residents of Recife, Brazil. Am J Public Health 2009;99:68-75.

33. Hallal PC, Reis RS, Hino AA et al. Avaliação de programas comunitários de promoção da atividade física: o caso de Curitiba, Paraná. Rev Bras Ativ Fis Saude 2009;14:104-13.

34. Venturim LM, Molina MC. Mudanças no estilo de vida após as ações realizadas no serviço de orientação ao exercício: Vitória/ES. Rev Bras Ativ Fis Saude 2005;10:4-16

35. Florindo AA, Hallal PC, Moura EC, Malta DC. Prática de atividades físicas e fatores associados em adultos, Brasil, 2006. Rev Saude Publica 2009;43(Supp 2):S65-73.

36. Salvador EP, Florindo AA, Reis RS, Costa EF. Perception of the environment and leisure-time physical activity in the elderly. Rev Saude Publica 2009;43:972-80.

37. Reis RS, Hino AA, Florindo AA, Añez CR, Domingues MR. Association between physical activity in parks and perceived environment: a study with adolescents. J Phys Act Health 2009;6:503-9.

38. Ippolito-Shepherd J. Escolas promotoras de saúde - fortalecimento da iniciativa regional: estratégias e linhas de ação 2003-2012. Washington: Organização Panamericana de Saúde, 2006.

39. Hoehner CM, Soares J, Parra Perez D et al. Physical activity interventions in Latin America: a systematic review. Am J Prev Med 2008;34:224-33.

40. Barros MV, Nahas MV, Hallal PC et al. Effectiveness of a school-based intervention on physical activity for high school students in Brazil: the Saúde na Boa project. J Phys Act Health 2009;6:163-9.

41. Foster GD, Sherman S, Borradaile KE et al. A policy-based school intervention to prevent overweight and obesity. Pediatrics 2008;121:e794-802.

42. Jaime PC, Lock K. Do school based food and nutrition policies improve diet and reduce obesity? Prev Med 2009:48:45-53.

43. Instituto Brasileiro de Geografia e Estatística. Pesquisa Nacional de Saúde do Escolar 2009. Rio de Janeiro: Instituto Brasileiro de Geografia e Estatística; 2010.

44. Hardman K, Marshall J. Second world-wide survey of school physical education. Alemanha: International Council of Sport Science and Physical Education, 2009.

45. Guedes JE, Guedes DP. Características dos programas de educação física escolar. Rev Paul Educ Fis 1997;11:49-62.

46. Guedes DP, Guedes JE. Esforços físicos nos programas de educação física escolar. Rev Paul Educ Fis 2001;15:33-44.

47. Guedes DP, Guedes JE. Educação física escolar: uma proposta de promoção da saúde. Revista da Associação dos Professores de Educação Física de Londrina 1993;8:16-23.

48. Nahas MV, Corbin CB. Educaçäo para a aptidão física e saúde: justificativa e sugestöes para implementaçäo nos programas de educação física. Rev Bras Cienc Mov 1992;6:14-24.

49. Pate RR, Hohn RC. Health and fitness through physical education. Champaign Human Kinetics, 1994.

50. Sallis JF, Conway TL, Prochaska JJ et al. The association of school environments with youth physical activity. Am J Public Health 2001;91:618-20.

51. Nielsen G, Taylor R, Williams S, Mann J. Permanent play facilities in school playgrounds as a determinant of children's activity. J Phys Act Health 2010;7:490-6.

52. Jones NR, Jones A, Sluijs EM et al. School environments and physical activity: the development and testing of an audit tool. Health Place 2010;16:776-83.

53. Ribeiro IC, Parra DC, Hoehner CM et al. School-based physical education programs: evidence-based physical activity interventions for youth in Latin America. Glob Health Promot 2010;17:5-15. 